\title{
Effects of Couple Stress and Porous Medium on Transient Magneto Peristaltic Flow under the Action of Heat Transfer
}

\author{
Ahmed M. Abdulhadi, Alaa Waleed Saleh \\ Department of Mathematics, College of Science, University of Baghdad,Baghdad, Iraq.
}

\begin{abstract}
Analytical and computational studies on transient peristaltic heat flow through a finite length porous channel are presented in this paper. the expression for temperature field, axial velocity, volume flow rate, pressure difference, local wall shear stress, stream function are obtained under the assumption of long wavelength and low Reynolds number. Effects of different physical parameters ,reflecting couple-stress parameter, permeability parameter, Hartman number, constant heat radiation factor and Grashof number, as well as amplitude ration on pumping characteristics and frictional force, stream lines pattern and trapping of peristaltic flow pattern studied with particular emphasis. The effect of heat transfer on two inherent phenomena of peristaltic flow is discussed numerically. This paper investigates the influence of MHD on a peristaltic flow of Newtonian fluid with couple stress through porous medium, where the no-slip assumption between wall and the fluid is no longer valid.
\end{abstract}

Keywords: Transient peristaltic flow, Heat transfer, Pressure gradient, Local wall shear stress , Couple stress, Magnetic field, Porous Medium.

\section{Introduction}

Peristalsis is a natural mechanism of pumping that is observed in the case of most physiological fluids. This behavior is usually associated with a progressive wave of area contraction or expansion along the length of the boundary of a fluid-filled distensible tube. This mechanism takes place in many practical appliances including roller and finger pumps, heart-lung machines ,blood pump machines, dialysis machines and also transport of noxious fluids in the nuclear and chemical waste industries .Owing to the importance of peristaltic flow, some significant investigation on this subject have been reported. Historically peristaltic fluid dynamics studies were initiated by (Shapiro et al.1969), who theoretically examined the peristaltic flow of viscous fluid induced by sinusoidal wall propagation. Heat transfer techniques change the internal energy of both systems involved and follow the first law of thermodynamics. Heat is generally transferred from objects of varying temperatures via conduction and convection. Several processes rely on heat transfer and the corresponding thermal coefficients. Several authors (T. Hayat, M.U. Qureshi 2010, Kh.S. Mekheimer, Y. Abd elmaboud 2008) have therefore reported the influence of heat transfer on peristaltic flow of Newtonian and non-Newtonian fluids (Reiner Rivlin fluid, Jeffrey-six constant fluid, second grade fluid, third order fluid, fourth grade fluid, Herschel Bulkley fluid and Johnson Segalman fluid) with or without the effect of magnetic field through uniform/nonuniform/asymmetric channels/vertical annulus systems and also porous media .

Further interesting studies include the recent papers by(Abd elmaboud and Mekheimer 2012) which considered thermal transport in transient flow in a vertical constricted annulus. A porous medium ( which contains a number of small holes distributed throughout the matter ) plays a key role in the study of transport process in bio- fluid mechanics, industrial mechanics, and engineering fields . A good example of peristaltic in porous medium is focused in intestinal fluid dynamics by (Y. Miyamoto et al.1983\&B.Jeffrey et al.2003) . Some authors (A.R. Rao, M. Mishra2004, T. Hayat, N. Ali, S. Asghar2007) studied peristaltic flow of Newtonian and non- Newtonian fluids such as power law fluid, magneto fluid and Maxwell fluid through the porous medium(T. Hayat, M.U. Qureshi, Q. Hussain2008),investigated the peristaltic flow with heat transfer in porous space (D. Tripathi ,O.A. Beg 2012)reported the influence of heat transfer on unsteady physiological magneto - fluid flow .( D. Tripathi2012). further studied the effect of heat transfer on peristaltic flow pattern through a finite length channel and discussed the impact of physical parameters on flow behavior, While he extended the peristaltic flow model in( D. Tripathi, 2013).for a finite porous channel and compare results with the results of( D. Tripathi2012).In view of the above discussion, in this paper, effect of heat transfer for nonNewtonian fluid with couple stress in MHD field through a porous medium with no slip boundary condition under the assumptions of long wave length and low Reynolds number. This transient couple stress peristaltic flow of Newtonian fluid through a finite length channel wall through porous medium..Intra-bolus pressure distribution, local wall shear stress and velocity profiles computed for the effects of the key hydrodynamic parameters .The influence of various pertinent parameters on the flow characteristics, Where study are discussed through graphs. 


\section{Mathematical Formulations}

Consider a transient peristaltic flow of viscous Newtonian fluid with couple - stress through a porous medium in uniform channel with heat transfer. Since we are considering uniform channel therefore the wall is maintained at temperature ( T1 ) and due to asymmetry at the center of the channel, the change of the temperature is taken to be zero ( $\mathrm{T} 0$ ) with constant speed $\mathrm{c}$ along the channel walls.

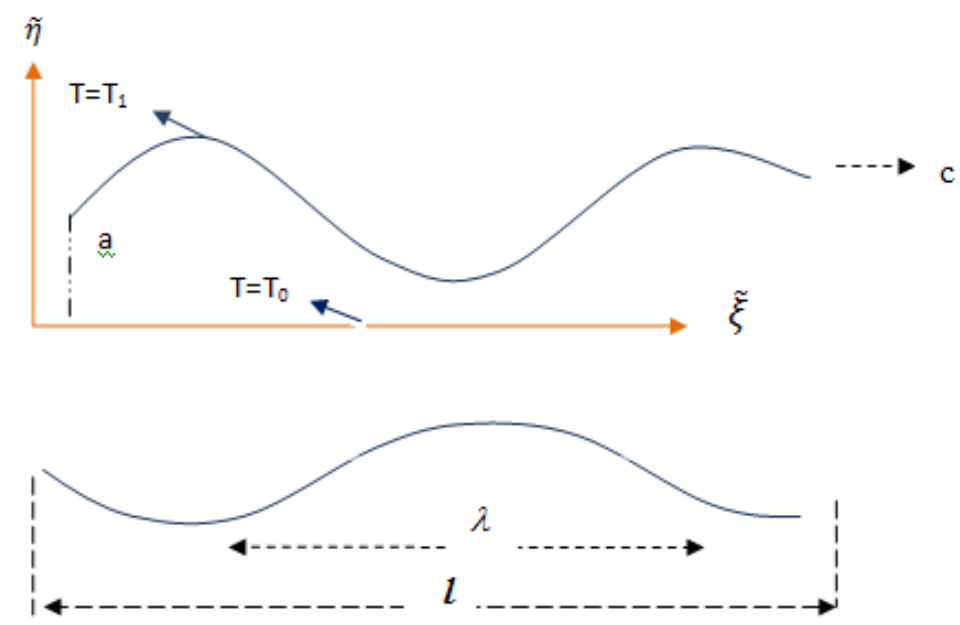

Fig(1): Geometry of the problem

The geometry of wall surface (see Fig.1) is described as :

$\tilde{h}(\tilde{\xi}, \tilde{t})=a-\tilde{\varphi} \cos ^{2} \frac{\pi}{\lambda}(\tilde{\xi}-c \tilde{t})$

Where $\tilde{h}, \tilde{\xi}, \tilde{t}, a, \tilde{\varphi}, \lambda$ and c represent the transverse vibration of the wall, the axial coordinate , time , the half width of the channel, the amplitude of the wave, the wavelength and the wave velocity respectively .

\section{III.Basic equation}

The basic equations governing the non-Newtonian fluid flow under the effect of a transverse magnetic field are given by :

The continuity equation :

$\frac{\partial \tilde{u}}{\partial \tilde{\xi}}+\frac{\partial \tilde{v}}{\partial \tilde{\eta}}=0$

The momentum equations are :

$$
\begin{aligned}
& \rho\left(\frac{\partial \tilde{u}}{\partial \tilde{t}}+\tilde{u} \frac{\partial \tilde{u}}{\partial \tilde{\xi}}+\tilde{v} \frac{\partial \tilde{v}}{\partial \tilde{\eta}}\right)=-\frac{\partial \tilde{p}}{\partial \tilde{\xi}}+\mu \nabla^{2} \tilde{u}-\mu_{1} \nabla^{4} \tilde{u}-\sigma B_{0}^{2} \tilde{u}-\frac{\mu}{\tilde{k}} \tilde{u} \\
& +\rho g \alpha\left(T-T_{0}\right) \\
& \rho\left(\frac{\partial \tilde{v}}{\partial \tilde{t}}+\tilde{u} \frac{\partial \tilde{v}}{\partial \tilde{\xi}}+\tilde{v} \frac{\partial \tilde{v}}{\partial \tilde{\eta}}\right)=-\frac{\partial \tilde{p}}{\partial \tilde{\eta}}+\mu \nabla^{2} \tilde{v}-\mu_{1} \nabla^{4} \tilde{v}-\sigma B_{0}^{2} \tilde{v}-\frac{\mu}{\tilde{k}} \tilde{v} \\
& \rho c_{p}\left(\frac{\partial}{\partial \tilde{t}}+\tilde{u} \frac{\partial}{\partial \tilde{\xi}}+\tilde{v} \frac{\partial}{\partial \tilde{\eta}}\right) T=k\left(\frac{\partial^{2} T}{\partial \tilde{\xi}^{2}}+\frac{\partial^{2} T}{\partial \tilde{\eta}^{2}}\right)+\Phi
\end{aligned}
$$


Where $\rho$ is the fluid density, $\tilde{u}$ axial velocity, $\tilde{v}$ transverse velocity, $\tilde{\eta}$ transverse coordinate $\tilde{p}$ pressure ,$\mu$ viscosity, $\mu_{1}$ constant associated with couple stress, $\sigma$ electrical conductivity, $B_{0}$ transverse magnetic field and $\tilde{k}$ permeability parameter $g$ acceleration due to gravity , $\alpha$ coefficient of linear thermal expansion of fluid, $\quad T$ temperature, $c_{p}$ specific heat at constant pressure and $\Phi$ constant heat addition / absorption. respectively .

$\nabla^{2}=\frac{\partial^{2}}{\partial \xi^{2}}+\frac{\partial^{2}}{\partial \eta^{2}} \quad, \quad \nabla^{4}=\frac{\partial^{4}}{\partial \xi^{4}}+\frac{\partial^{4}}{\partial \eta^{4}}+2 \frac{\partial^{4}}{\partial \xi^{2} \partial \eta^{2}}$

In order to simplify the governing equations of the motion , We introducing the following dimensionless parameter :

$$
\left.\begin{array}{l}
\xi=\frac{\tilde{\xi}}{\lambda}, \eta=\frac{\tilde{\eta}}{a}, u=\frac{\tilde{u}}{c}, v=\frac{\tilde{v}}{c \delta}, \delta=\frac{a}{\lambda}, t=\frac{c \tilde{t}}{\lambda}, K=\frac{\tilde{k}}{a^{2}} \\
p=\frac{\tilde{p} a^{2}}{\mu c \lambda}, \alpha=a \sqrt{\frac{\mu}{\mu_{1}}}, M=\sqrt{\frac{\sigma}{\mu}} a B_{0}, h=\frac{\tilde{h}}{a}, P_{r}=\frac{\mu c p}{k} \\
G_{r}=\frac{\rho \alpha g a^{2}\left(T_{1}-T_{0}\right)}{\mu c}, \theta=\frac{\left(T-T_{0}\right)}{\left(T_{1}-T_{0}\right)}, \beta=\frac{a^{2} \Phi}{k\left(T_{1}-T_{0}\right)}, \operatorname{Re}=\frac{\rho c a}{\mu}
\end{array}\right\}
$$

Where $(\delta)$ is the wave number, $(\operatorname{Re})$ Reynolds number , $(\alpha)$ is the couple stress parameter, $(M)$ is the Hartmann number, $(K)$ is the thermal conductivity, $\left(G_{r}\right)$ Grashof number, $\left(P_{r}\right)$ Prandtle number, $(\theta)$ temperature distribution, and $(\beta)$ constant heat radiation .Substituting (6) into equations ( 1 ) - ( 5 ), we obtain the following non-dimensional equations and boundary conditions :

$$
\begin{aligned}
& h(\xi, t)=1-\varphi \cos ^{2} \pi(\xi, t) \\
& \frac{\partial u}{\partial \xi}+\frac{\partial v}{\partial \eta}=0 \text {. } \\
& \operatorname{Re} \delta\left(\frac{\partial u}{\partial t}+u \frac{\partial u}{\partial \xi}+v \frac{\partial u}{\partial \eta}\right)=-\frac{\partial p}{\partial \xi}+\left(\delta^{2} \frac{\partial^{2}}{\partial \xi^{2}}+\frac{\partial^{2}}{\partial \eta^{2}}\right) u- \\
& \frac{1}{\alpha^{2}}\left(\delta^{4} \frac{\partial^{4}}{\partial \xi^{4}}+\frac{\partial^{4}}{\partial \eta^{4}}+2 \delta^{2} \frac{\partial^{4}}{\partial \xi^{2} \partial \eta^{2}}\right) u- \\
& M^{2} u-\frac{1}{k} u+G_{r} \theta \\
& \operatorname{Re} \delta^{3}\left(\frac{\partial v}{\partial t}+u \frac{\partial v}{\partial \xi}+v \frac{\partial v}{\partial \eta}\right)=-\frac{\partial p}{\partial \eta}+\delta^{4} v c \frac{\partial^{4} u}{\partial \eta^{4}}+\delta^{2} v c \frac{\partial^{2} u}{\partial \eta^{2}}-\delta^{4} \frac{\mu_{1}}{\mu \lambda^{2}} v c \\
& \frac{\partial^{4} u}{\partial \xi^{4}} \delta \frac{\mu_{1}}{\mu} v c \frac{\partial^{4} u}{\partial \eta^{4}}-2 \delta \frac{\mu_{1}}{\mu} \frac{a}{\lambda^{3}} v \frac{\partial^{4} u}{\partial \xi^{2} \partial \eta^{2}} \\
& -\delta \frac{\mu}{k a^{2}} v c
\end{aligned}
$$

$\operatorname{Re} P_{r} \delta\left(\frac{\partial}{\partial t}+u \frac{\partial}{\partial \xi}+v \delta \frac{\partial}{\partial \eta}\right) \theta=\left(\delta^{2} \frac{\partial^{2}}{\partial \xi^{2}}+\frac{\partial^{2}}{\partial \eta^{2}}\right) \theta+\beta$

The above problem, will be solve subject to the following boundary conditions : 
(no slip condition)

(the regularity condition)

( the vanishing of couple stresses) $\frac{\partial^{2} u}{\partial \eta^{2}}=0$ at $\eta=\mathrm{h}, \frac{\partial^{3} u}{\partial \eta^{3}}=0$ at $\eta=0$

(finite length conditions)

(the temperature conditions)

$$
\begin{aligned}
& p(\xi, t)=0 \text { at } \xi=0, p(\xi, t)=0 \text { at } \xi=l \\
& \theta=0 \text { at } \eta=0, \theta=1 \text { at } \eta=h
\end{aligned}
$$

\section{Solution of the problem}

The general solution of the governing equations ( 8 ) - (11) in the general case seems to be impossible ; therefore, we shall confine the analysis under the assumption of small dimensionless wave number. It follows that $\delta \ll 1$. In other words, we considered the long - wavelength approximation. Along to this assumption, equations (8) - (11) become :

$$
\begin{aligned}
& \frac{\partial u}{\partial \xi}+\frac{\partial v}{\partial \eta}=0 \\
& \frac{\partial p}{\partial \xi}=\frac{\partial^{2} u}{\partial \eta^{2}}-\frac{1}{\alpha^{2}} \frac{\partial^{4} u}{\partial \eta^{4}}-N^{2} u+G_{r} \theta
\end{aligned}
$$$$
\frac{\partial p}{\partial \eta}=0
$$

$$
\frac{\partial^{2} \theta}{\partial \eta^{2}}+\beta=0
$$

Where $N^{2}=M^{2}+\frac{1}{K}$,

The solution of Eq. ( 20 ) subject to the associated boundary conditions (16) is found of the form ..

$\theta=\frac{\eta}{h}+\frac{\beta}{2}\left(h \eta-\eta^{2}\right)$

Substituted in Eq.(18) and the solution of Eq. (18) subject to the associated boundary condition is found of the form

$$
\begin{gathered}
u=\left(\frac{2 \beta G_{r} h-2 \alpha^{2} h N^{2} \partial p / \partial \xi-2 G_{r} N^{2} \eta-\beta G_{r} h^{2} N^{2} \eta+\beta G_{r} h N^{2} \eta^{2}}{2 \alpha^{2} h N^{4}}\right) \\
\left(\frac{1}{\left(m_{1}^{2}-m_{2}^{2}\right)}\left\{\frac{m_{2}^{2} \cosh \left[m_{1} \eta\right]}{\cosh \left[m_{1} h\right]}-\frac{m_{1}^{2} \cosh \left[m_{2} \eta\right]}{\cosh \left[m_{2} h\right]}\right\}+1\right)
\end{gathered}
$$

Where

$$
m_{1}=\sqrt{\frac{\alpha^{2}+\alpha \sqrt{\alpha^{2}-4 N^{2}}}{2}} \quad, \quad m 2=\sqrt{\frac{\alpha^{2}-\alpha \sqrt{\alpha^{2}-4 N^{2}}}{2}}
$$


The local wall shear stress defined as $\tau_{w}=\left.\frac{\partial u}{\partial \eta}\right|_{\eta=h}$, further by using Eq.(22),

$\tau_{w}=\left(\frac{2 \beta G_{r} h-2 G_{r} h N^{2}\left\{m_{1} m_{2}^{2} \tanh \left[m_{1} h\right]-m_{2} m_{1}^{2} \tanh \left[m_{2} h\right]\right\}}{2 \alpha^{2} h N^{4}\left(m_{1}^{2}-m_{2}^{2}\right)}\right)$

Volume flow rate, an important parameter in channel flows, is defined as $Q(\xi, t)=\int_{0}^{h} u d \eta$, which on integrating Eq. (22) yields :

$$
\begin{aligned}
& Q=\left(\frac{2 \beta G_{r} h-2 \alpha^{2} h N^{2} \partial p / \partial \xi-G_{r} N^{2} \eta-\frac{\beta G_{r} h^{3} N^{2}}{2}+\beta G_{r} h N^{2} \frac{h^{3}}{3}}{2 \alpha^{2} h N^{4}}\right) \\
& \left(\frac{1}{\left(m_{1}^{2}-m_{2}^{2}\right)}\left\{m_{1} m_{2}^{2} \tanh \left[m_{1} h\right]-m_{2} m_{1}^{2} \tanh \left[m_{2} h\right]\right\}+h\right)
\end{aligned}
$$

The following expression define the existing relation between the averaged flow rate and flow rate in the wave frame and in the laboratory frame :

$$
\tilde{Q}=q+1-\frac{\varphi}{2}=Q-h+1-\frac{\varphi}{2}
$$

A simple manipulation of Eq. (24), followed by application of Eq. (25), yields the pressure gradient as follows

$$
\begin{aligned}
& \frac{\partial p}{\partial \xi}=-N^{2}\left(\frac{\tilde{Q}+h-1+\frac{\varphi}{2}}{h f}-\frac{\beta G_{r}}{\alpha^{2} N^{4}}+\frac{\beta G_{r} h^{2}}{12 \alpha^{2} N^{2}}+\frac{G_{r}}{2 \alpha^{2} N^{2}}\right) \\
& f=\left(\frac{1}{\left(m_{1}^{2}-m_{2}^{2}\right)}\left\{m_{1} m_{2}^{2} \tanh \left[m_{1} h\right]-m_{2} m_{1}^{2} \tanh \left[m_{2} h\right]\right\}+h\right)
\end{aligned}
$$

Which, on integration between 0 and $\xi$, generates the following expression for pressure difference :

$$
p(\xi, t)-p(0, t)=\int_{0}^{\xi}\left[-N^{2}\left(\frac{\widetilde{Q}+h-1+\frac{\varphi}{2}}{h f}-\frac{\beta G_{r}}{\alpha^{2} N^{4}}+\frac{\beta G_{r} h^{2}}{12 \alpha^{2} N^{2}}+\frac{G_{r}}{2 \alpha^{2} N^{2}}\right)\right] d s
$$

The pressure difference $\left(\Delta p_{l}\right)$, across the one wavelength ,which upon utilizing Eq.(26), assumes the form :

$$
\Delta p_{l}=\int_{0}^{\xi} \frac{\partial p}{\partial \xi} d \xi
$$

Stream function can be obtained by integrating equation (22) with respect to $\eta$, and using Eq. (26) 


$$
\begin{gathered}
\psi=\left(\frac{\beta G_{r} h\left(\frac{1}{6} h^{2} \eta-\frac{1}{2} h \eta+\frac{1}{3} \eta^{3}\right)+G_{r}\left(h \eta-\eta^{2}\right)+\frac{2}{f} \alpha^{2} N^{2} Q \eta}{2 \alpha^{2} h N^{2}}\right) \\
\left(\frac{1}{\left(m_{1}^{2}-m_{2}^{2}\right)}\left(\frac{m_{1} m_{2}^{2} \sinh \left[m_{1} \eta\right]}{\cosh \left[m_{1} h\right]}-\frac{m_{2} m_{1}^{2} \sinh \left[m_{2} \eta\right]}{\cosh \left[m_{2} h\right]}\right)+\eta\right)
\end{gathered}
$$

\section{Numerical Results and Discussion}

In this section, the numerical and computational results are discussed for the problem of couple-stress Newtonian fluid in a tube with porous medium through the graphical illustrations. The transient magneto peristaltic flow of couple-stress fluids through the porous medium with effect of heat transfer are discussed through Figures (2-17). MATHEMATICA program is used to find out numerical results and illustrations.

A. Based on Eq. 15 , Figs.(2-5) illustrates the effects of the parameters time (t), Grashof number $\left(G_{r}\right)$, constant heat radiation $(\beta)$ and permeability parameter $(k)$ on the axial velocity distribution, respectively. Figures show the relation between axial velocity and transverse displacement. It is observed that the axial velocity profile region increases with increasing magnitudes of $\left(G_{r}\right)$ and $(\beta)$ While velocity decrease when the other parameters increase, finally noticed that the velocity take the a trough located and will be minimized at the channel center .

B. Figs. (6-9) shows the influence of heat transfer on local wall shear stress with axial distance along the length of the channel at various instant .The relation between local shear stress and axial distance is nonlinear where the curves exhibit parabolic shape .

C. Figs. (10-13) illustrate the relation between pressure difference across one wavelength and averaged volume flow rate, that exhibits a linear relation between them. for the effect of $\mathrm{t},\left(G_{r}\right),(\beta)$ and $(k)$. volume flow rate increase with increasing the magnitude of Grashof number $\left(G_{r}\right)$,constant heat radiation $(\beta)$.

D. Figs.(14-16) illustrate the pressure distribution along the length of channel at various instants, for the influence of $\left(G_{r}\right),(\beta)$ and $(k)$ at various times whereas $l=2$.It is physically interpreted that if heat transfer is effective then less pressure is required for the peristaltic flow of viscous fluid through the channel .

E. Figs.17,18,19drawn for streamline patterns. The impacts of Grashof parameter, constant heat parameter, couple- stress parameter, Hartman parameter, permeability parameter and time are discussed through these figures. It is important to observe that the size of trapping bolus reduces when the magnitude of said parameters $\left(\left(G_{r}\right),(\beta),(\alpha),(M),(k)\right.$ and $\left.(t)\right)$ increases.

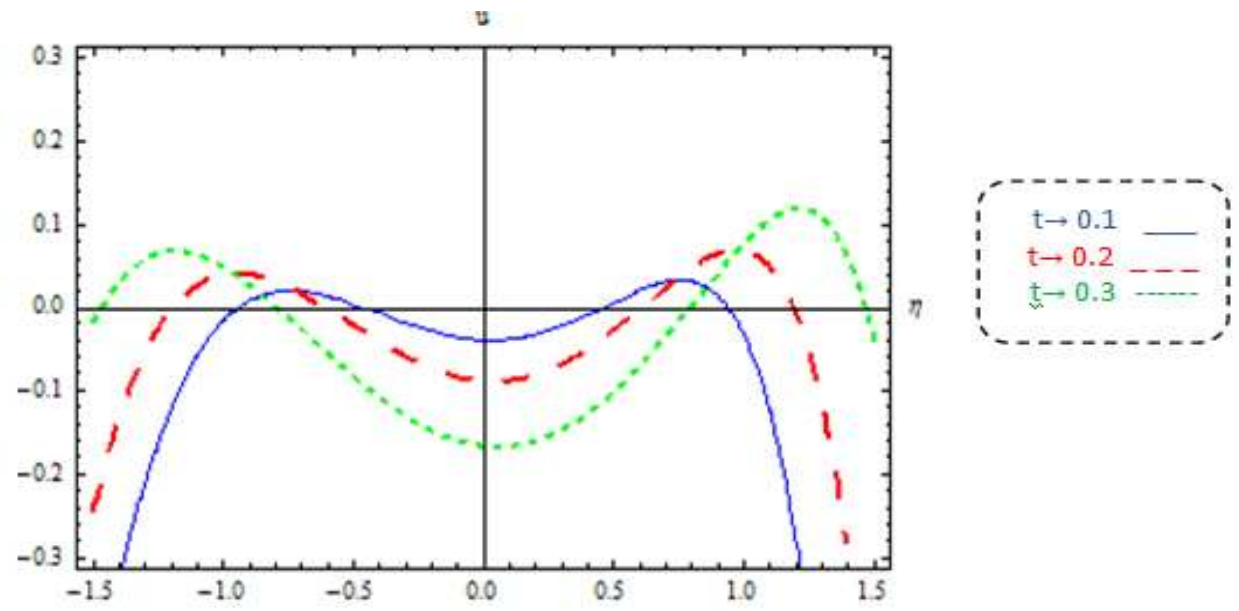

Fig 2. The axial velocity vs. transverse displacement for different values 
of $t$ with $\xi=1, \frac{\partial p}{\partial \xi}=1, M=1, \alpha=3, \varphi=0.6, k=5, G_{r}=1, \beta=1$.

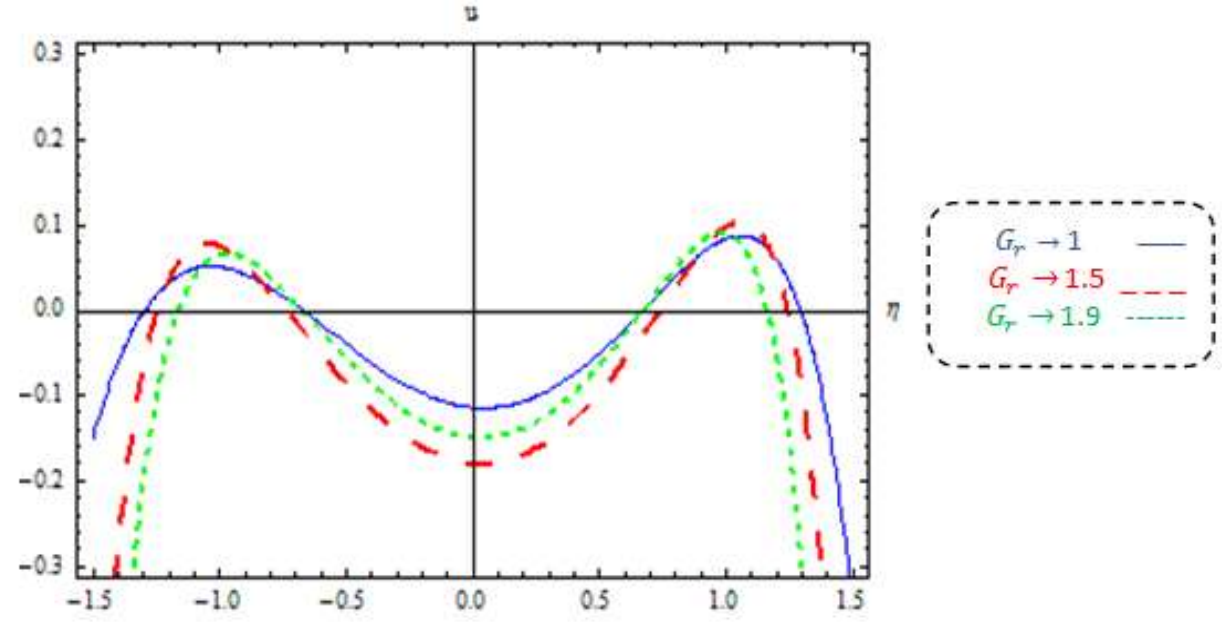

Fig 3. The axial velocity vs. transverse displacement for different values of $G_{r}$ with $\xi=1, \frac{\partial p}{\partial \xi}=1, M=1, \alpha=3, \varphi=0.6, k=5, t=0.2, \beta=1$

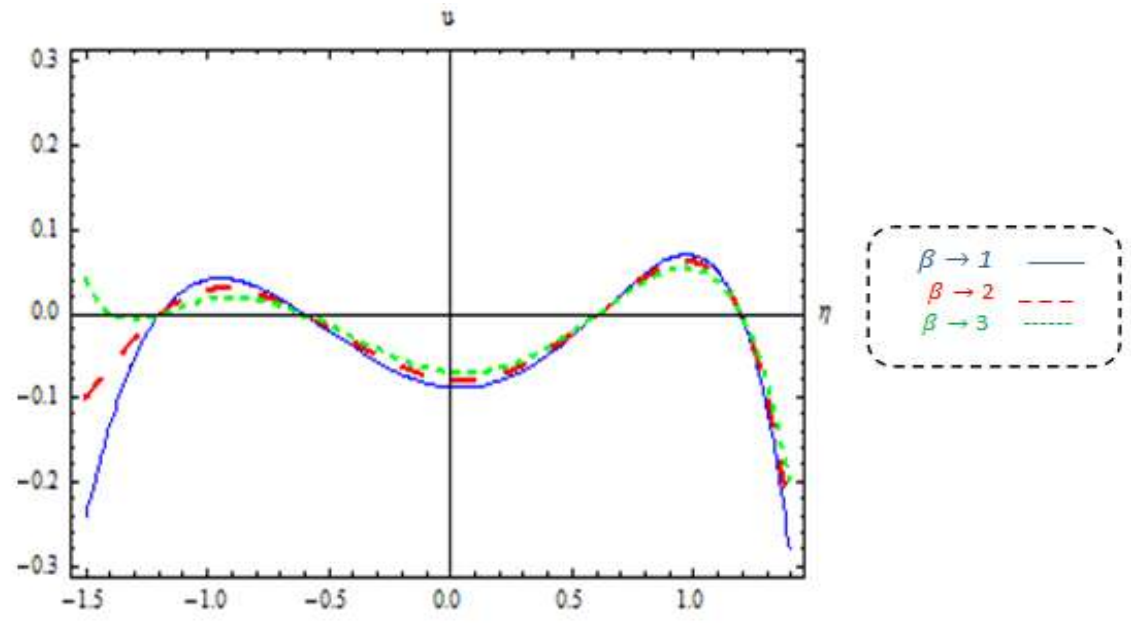

Fig 4. The axial velocity vs. transverse displacement for different values of $\beta$ with $\xi=1, \frac{\partial p}{\partial \xi}=1, M=1, \alpha=3, \varphi=0.6, k=5, t=0.2, G_{r}=1$

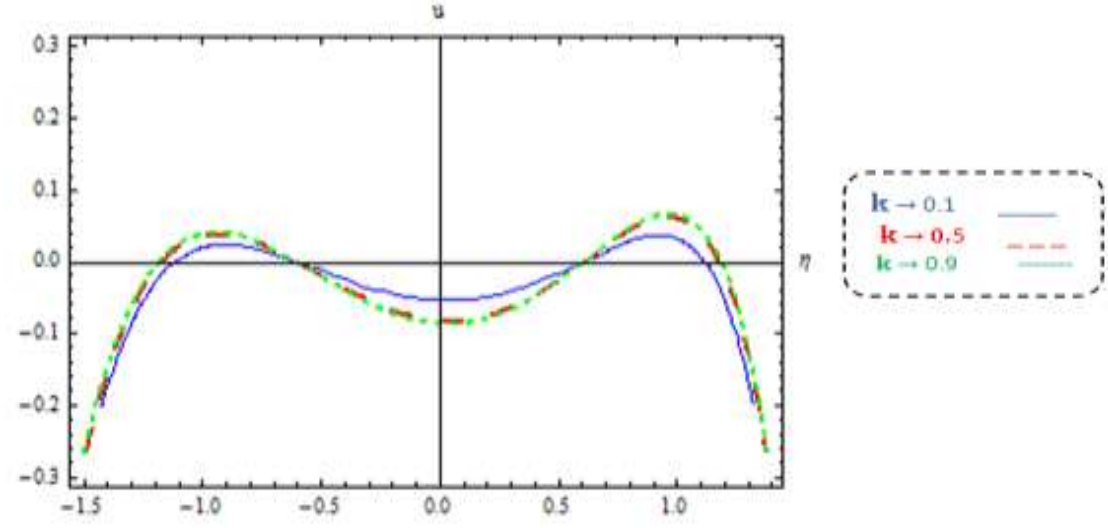

Fig 5. The axial velocity vs. transverse displacement for different values 
of $k$ with $\xi=1, \frac{\partial p}{\partial \xi}=1, M=1, \alpha=3, \varphi=0.6, G_{r}=1, t=0.2, \beta=1$

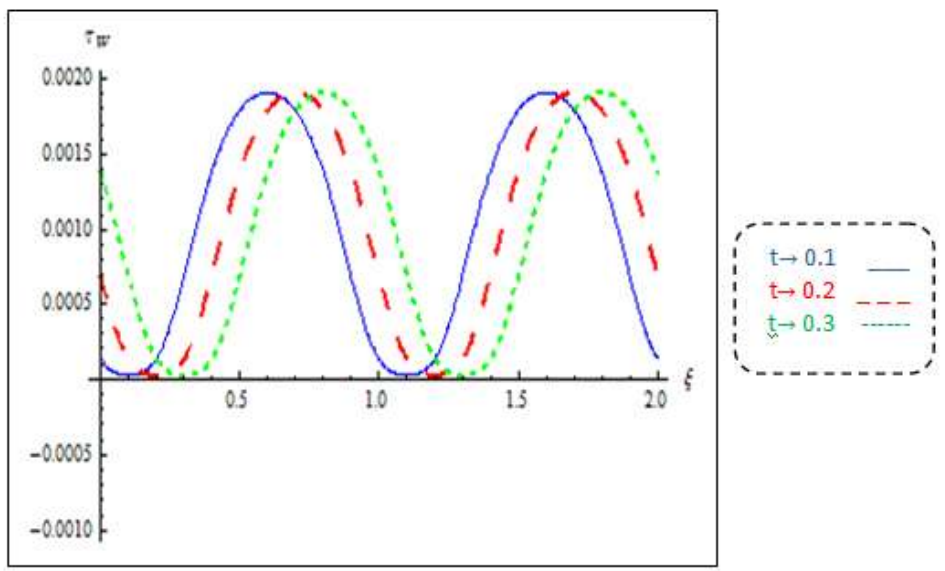

Fig 6. Local wall shear stress vs. axial distance for different values of $t$ with $\beta=2, G_{r}=1, M=1, l=2, \alpha=3, \varphi=0.9, k=5$.
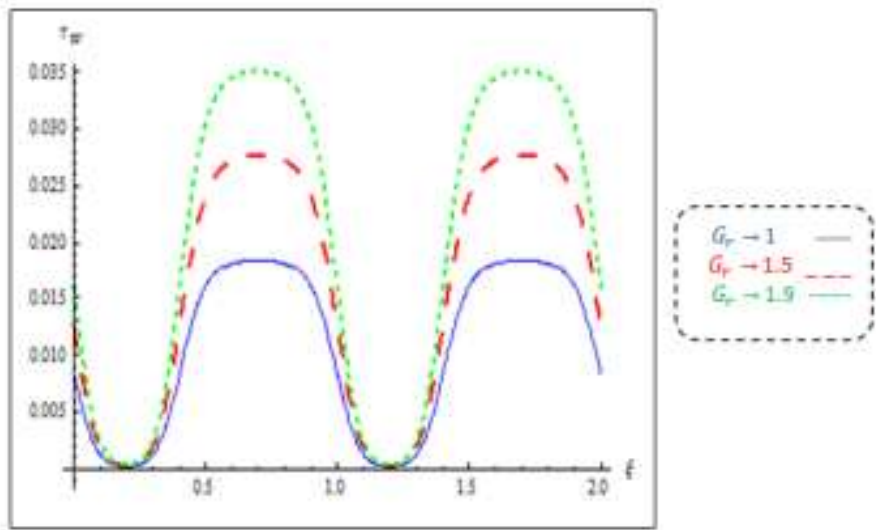

Fig 7. Local wall shear stress vs. axial distance for different values of $G_{r}$ with $\beta=2, t=0.2, M=1, l=2, \alpha=3, \varphi=0.9, k=5$.
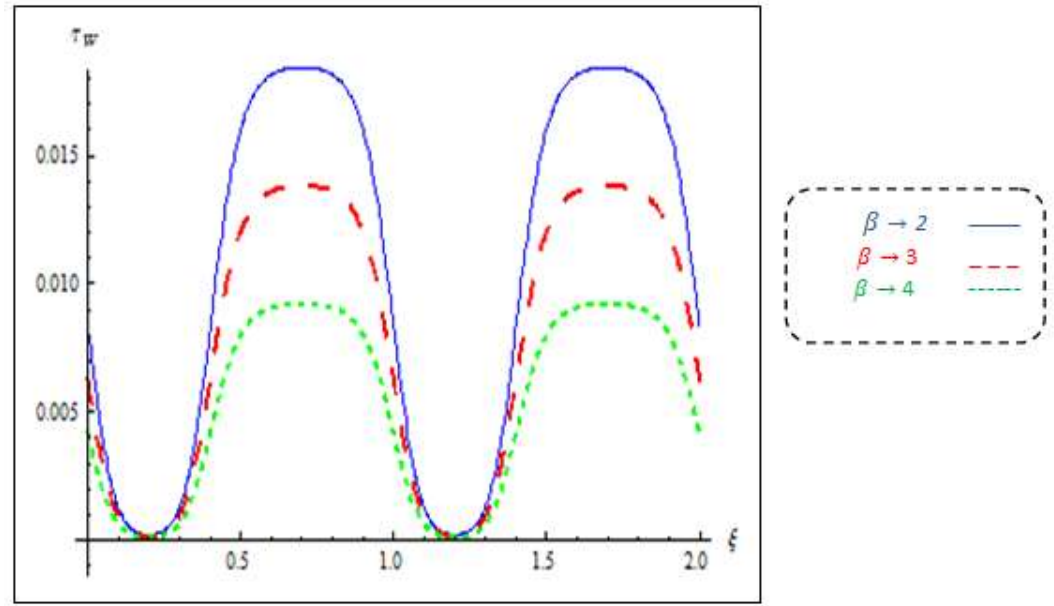

Fig 8. Local wall shear stress vs. axial distance for different values of $\beta$ with $t=0.2, G_{r}=1, M=1, l=2, \alpha=3, \varphi=0.9, k=5$. 


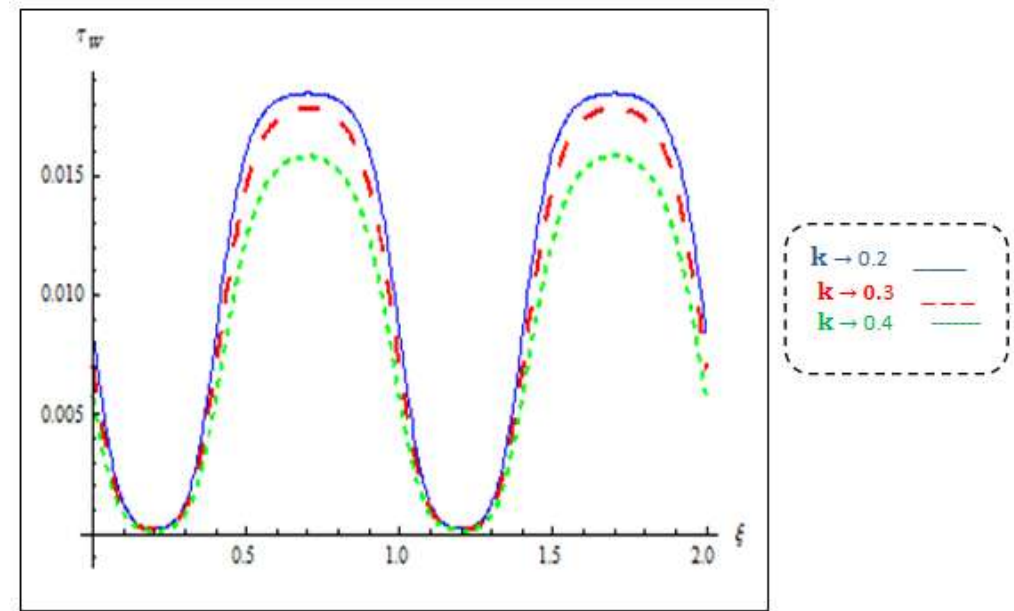

Fig 9. Local wall shear stress vs. axial distance for different values of $k$ with $\beta=2, G_{r}=1, M=1, l=2, \alpha=3, \varphi=0.9, t=0.2$.
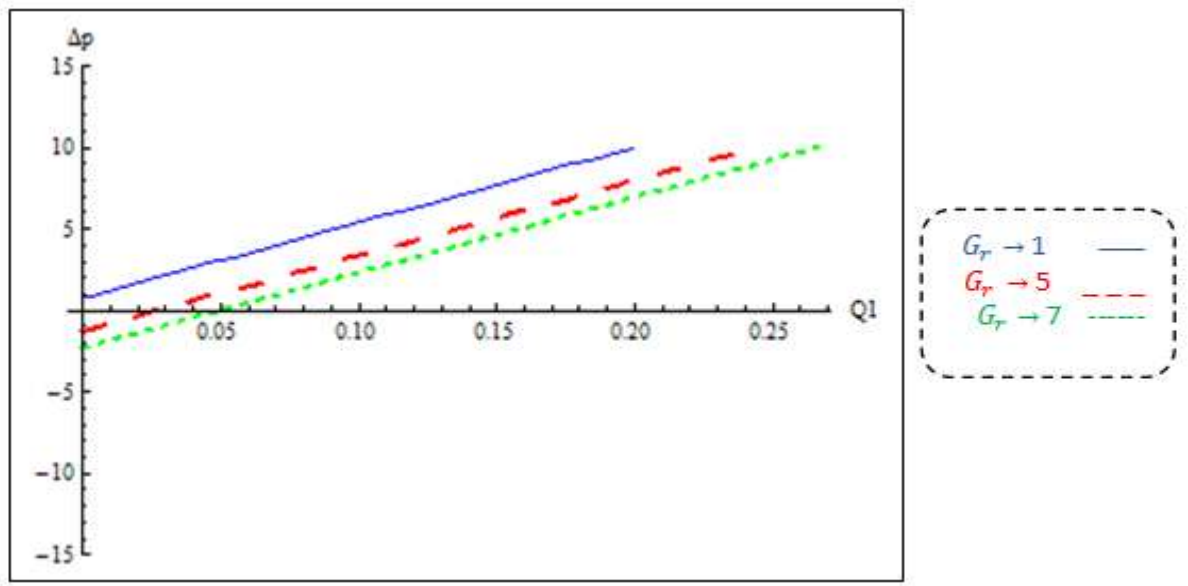

Fig 10. The pressure difference vs. averaged flow rate for different values of $G_{r}$ with $\alpha=1, \beta=1, l=1, \varphi=0.01, t=2, M=1, k=0.1$.

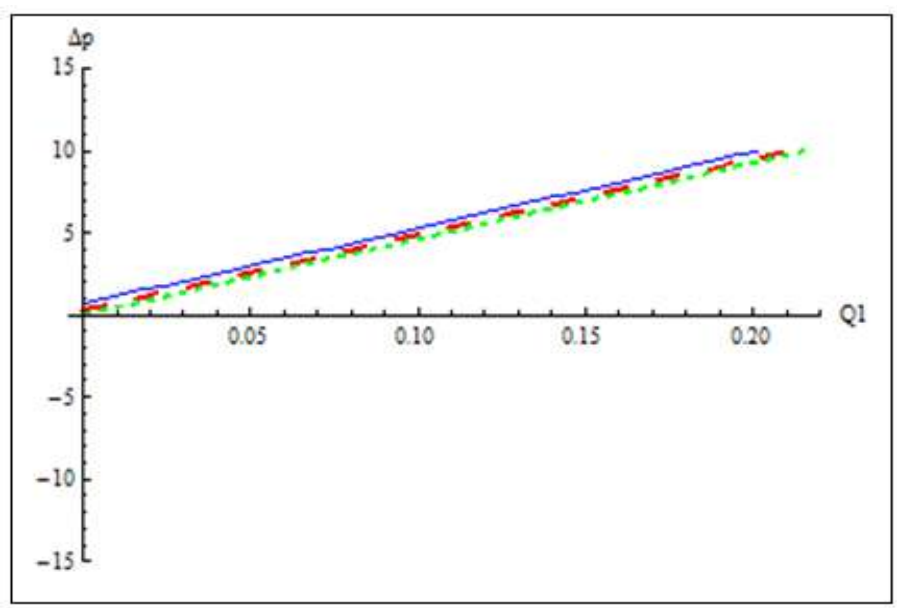

$$
\begin{aligned}
& \beta \rightarrow 1 \\
& \beta \rightarrow 5 \\
& \beta \rightarrow 7
\end{aligned}
$$

Fig 11. The pressure difference vs. averaged flow rate for different values of $\beta$ with $\alpha=1, G_{r}=1, l=1, \varphi=0.01, t=2, M=1, k=0.1$. 

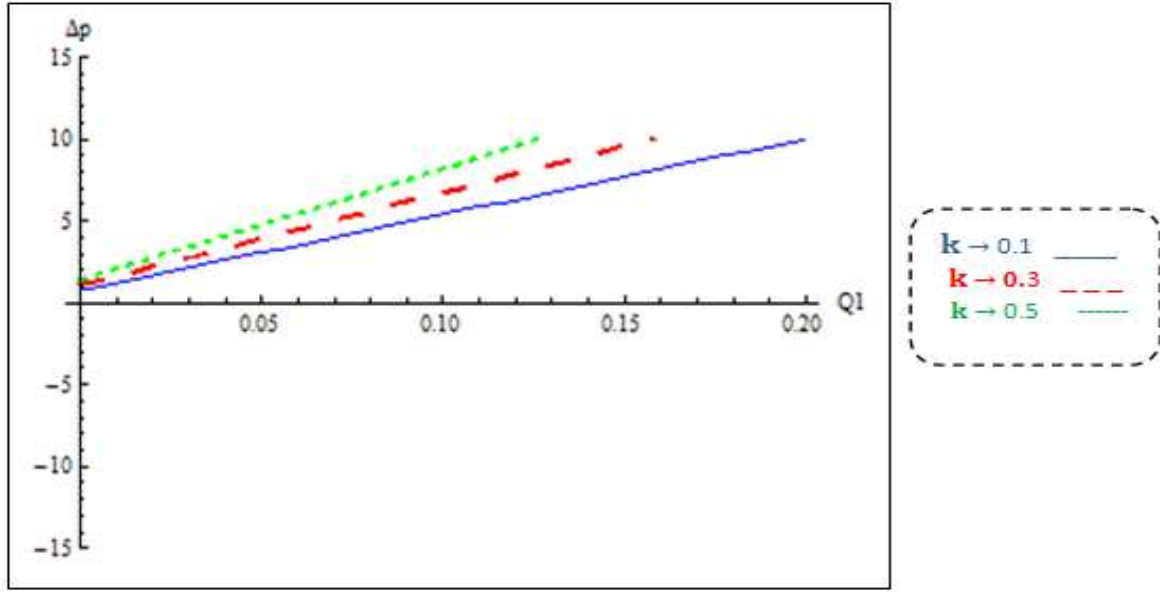

Fig 12. The pressure difference vs. averaged flow rate for different values of $k$ with $\alpha=1, G_{r}=1, \beta=1, l=1, \varphi=0.01, t=2, M=1$.
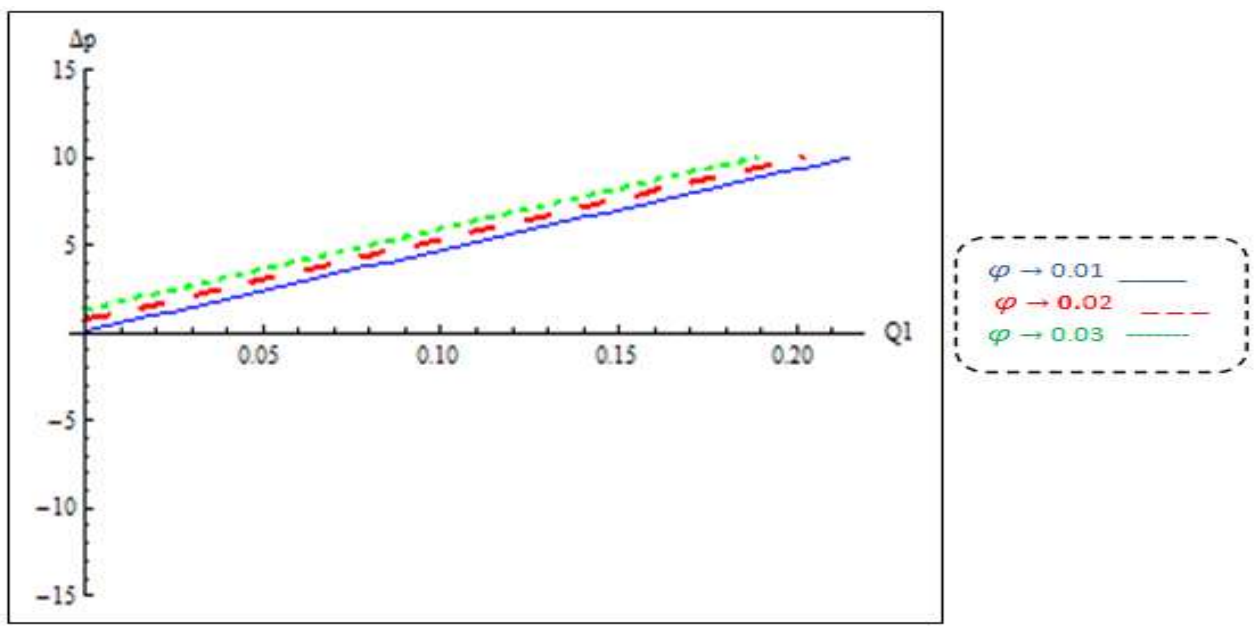

Fig 13. The pressure difference vs. averaged flow rate for different values of $\varphi$ with $\alpha=1, G_{r}=1, \beta=1, l=1, t=2, M=1, k=0.1$.
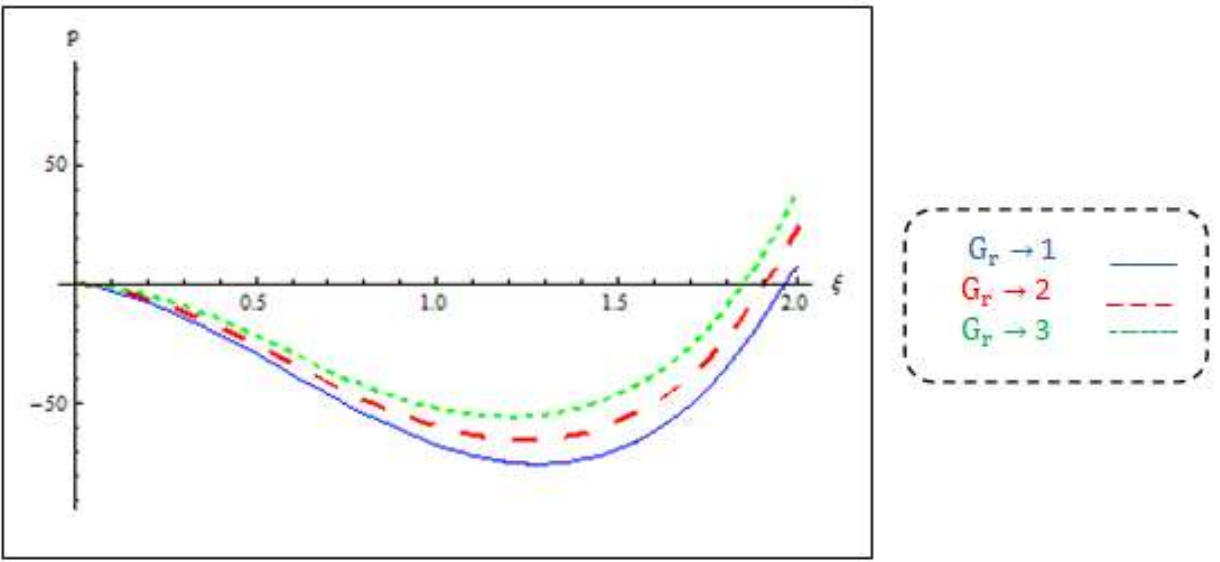

Fig 14. The pressure distribution vs. axial distance for different values of $G_{r}$ with $\alpha=10, \beta=1, t=0.2, M=1, \tilde{Q}=0.95, \varphi=0.9, k=5$. 

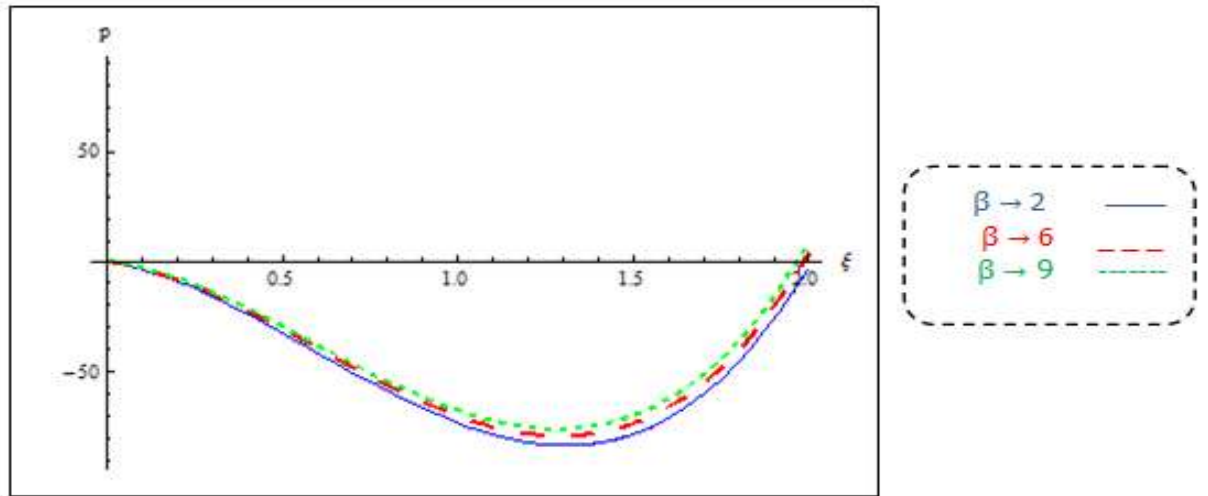

Fig 15. The pressure distribution vs. axial distance for different values of $\beta$ with $\alpha=10, G_{r}=1, t=0.2, M=1, \tilde{Q}=0.95, \varphi=0.9, k=5$.

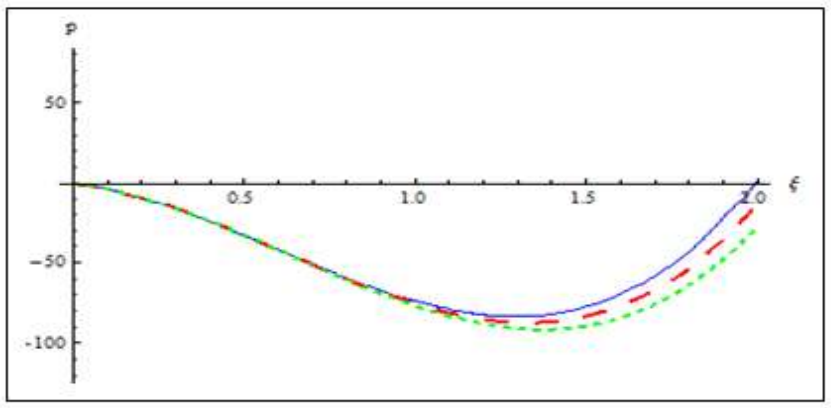

$\mathbf{k} \rightarrow 5.21$

$k \rightarrow 5.23$

$\mathrm{k} \rightarrow 5.25$

Fig 16. The pressure distribution vs. axial distance for different values of $k \alpha=10, G_{r}=1, \beta=1, t=0.2, M=1, \widetilde{Q}=0.95, \varphi=0.9$.

(a)

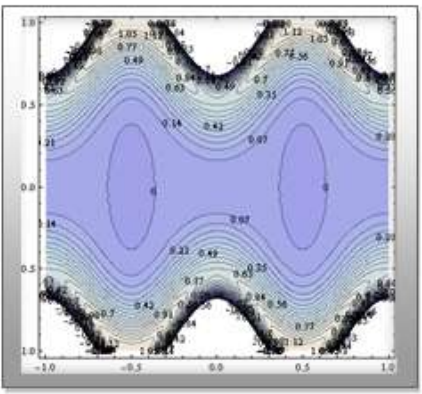

(b)

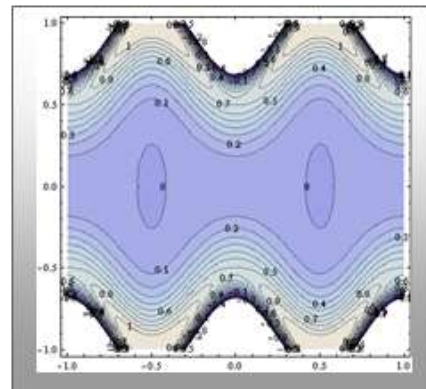

(c)

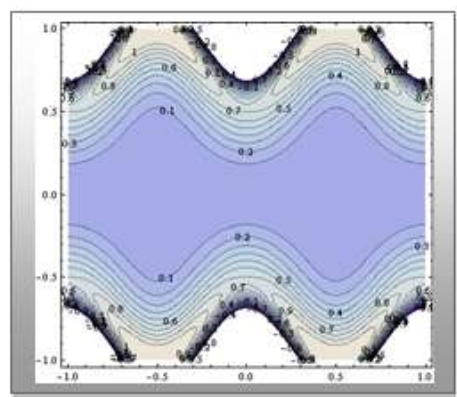

Fig 17. Streamline in the wave frame at $\tilde{Q}=0.95 \& \varphi=0.5$ When

(a) $k=0.1, G_{r}=1, \alpha=10, \beta=1, t=1, M=2$

(b) $k=0.16, G_{r}=1, \alpha=10, \beta=1, t=1, M=2$ 
(c) $k=0.19, G_{r}=1, \alpha=10, \beta=1, t=1, M=2$

(a)

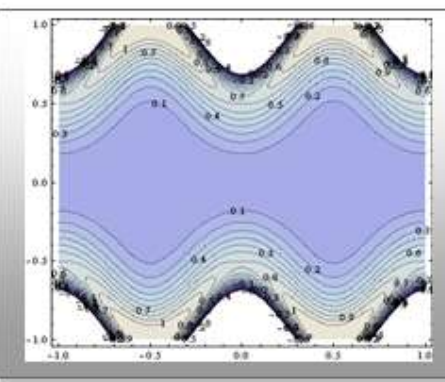

(b)

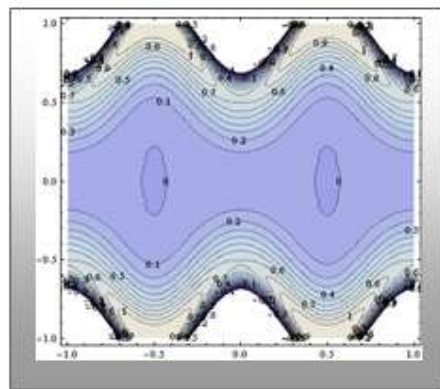

(c)

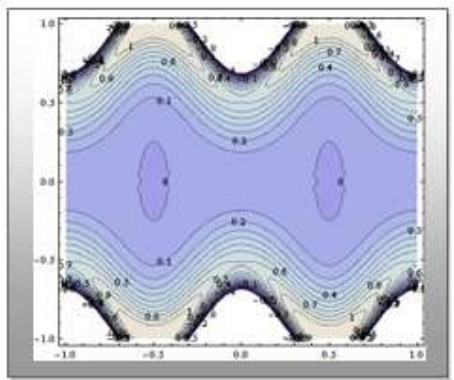

Fig 18. Streamline in the wave frame at $\tilde{Q}=0.95 \& \varphi=0.5$ When
(a) $G_{r}=3, \beta=1, \alpha=10, k=0.3, M=2, t=1$
(b) $G_{r}=6, \beta=1, \alpha=10, k=0.3, M=2, t=1$
(c) $G_{r}=9, \beta=1, \alpha=10, k=0.3, M=2, t=1$

(4)

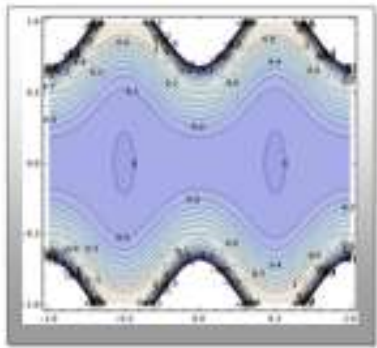

(b)

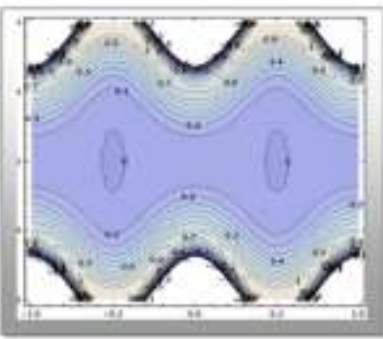

(4)

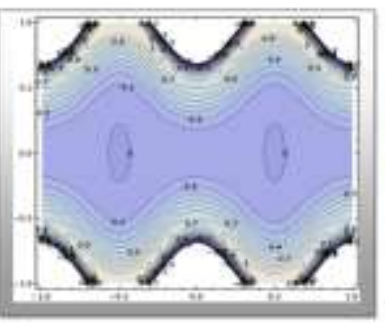

Fig 19. Streamline in the wave frame at $\tilde{Q}=0.95 \& \varphi=0.5$ When
(a) $\beta=2, G_{r}=3, \alpha=10, k=0.3, t=1, M=2$
(b) $\beta=4, G_{r}=3, \alpha=10, k=0.3, t=1, M=2$
(c) $\beta=8, G_{r}=3, \alpha=10, k=0.3, t=1, M=2$ 


\section{Concluding remarks}

we have discussed the influence of heat transfer on transient magneto peristaltic flow with couple-stress fluid Through porous medium on a Newtonian fluid in a finite length channel under a transverse magnetic field . The results are discussed through graphs. We have concluded the following observations:

1. The axial velocities increase with the increase in $\left(G_{r}\right),(\beta)$ and decrease with the increase in $(t),(k)$.

2. The local wall shear stress decrease with increasing in $\mathrm{k}$ and $(\beta)$.

3. The increasing of Grashof number $\left(G_{r}\right)$ time(t) reduces local wall shear stress at all distances along the channel axis with decrease on it .

4. the pressure difference decrease with increasing in $k$ and $\varphi$ where as it increase with increasing in $\left(G_{r}\right)$ and $(\beta)$.

5. A linear relationship is computed between axial pressure difference and volume flow rate.

6. 6 .The pressure distribution decreases with the increasing in $(\beta)$ and $\left(G_{r}\right)$ whereas it displaced further along the channel axis with increasing in $k$.

7. the decreasing in length of the finite channel has a major effect on the pressure distribution and in some cases on magnitudes .

8. Finally, it is concluded that the trapping enhances with increasing the effect of porosity, whereas it reduces with increasing the effect of heat transfer

\section{References}

[1]. A.H. Shapiro, M.Y. Jaffrin, S.L. Weinberg, (1969).Peristaltic pumping with long wavelengths at low Reynolds number, Journal of Fluid Mechanics 37 ,799-825.

[2]. A.R. Rao, M. Mishra, Peristaltic transport of a power-law fluid in a porous tube, (2004). Journal of Non-Newtonian Fluid Mechanics121,163-174.

[3]. B. Jeffrey, H.S. Udaykumar, K.S. Schulze,(2003).Flow fields generated by peristaltic reflex in isolated guinea pig ileum: impact of contraction depth and shoulders ,American Journal of Physiology-Gastrointestinal and Liver Physiology 285 , G907 - G918 .

[4]. D. Tripathi,O. A. Beg ,(2012). A study of unsteady physiological magneto-fluid flow and heat transfer through a finite length channel by peristaltic pumping Proceedings of the Institution of Mechanical Engineers ,Part H: Journal of Engineering in Medicine 226, 631-644.

[5]. D. Tripathi ,(2012). A mathematical model for swallowing of food bolus through the oesophagus under the influence of heat transfer, International Journal of Thermal Science 51, $91-101$.

[6]. D. Tripathi, (2013).Study of transient peristaltic flow through a finite porous channel, Mathematical and Computer Modelling 57 , $1270-1283$.

[7]. G. Radhakrishnamacharya, C. Srinivasulu, (2007). Influence of wall properties on peristaltic transport with heat transfer, Comptes Rendus Mecanique 335, 369-373.

[8]. Kh.S. Mekheimer, Y. Abd elmaboud, (2008). The influence of heat transfer and magnetic field on peristaltic transport of a Newtonian fluid in a vertical annulus:

Application of endoscope, Physics Letters A 372, 1657-1665.

[9]. Kh.S. Mekheimer, Nonlinear peristaltic transport through a porous medium in an inclined planar channel ,(2003).Journal of Porous Media 6, 189-201.

[10]. K. Vajravelu, G. Radhakrishnamacharya, V.Radhakrishnamurty,(2007). Peristaltic transport and heat transfer in a vertical porous annulus with long wave approximation, International Journal of Non-Linear Mechanics 42 ,754-759.

[11]. M. Kothandapani, S. Srinivas, (2008). On the influence of wall properties in the MHD peristaltic transport with heat transfer and porous medium, Physics Letters A372,4586 - 4591.

[12]. N.S. Akbar, S. Nadeem, (2010).Simulation of heat and chemical reactions on Reiner Rivlin fluid model for blood flow through a tapered artery with a stenosis, Heat and Mass Transfer 46, 531 - 539.

[13]. S. Nadeem, N.S. Akbar, M. Hameed, (2010).Peristaltic transport and heat transfer of a MHD Newtonian fluid with variable viscosity, International Journal for Numerical Methods in Fluids 63, 1375-1393.

[14]. S. Nadeem, N.S. Akbar, (2010). Simulation of second grade fluid model for blood flow through a tapered artery with a stenosis, Chinise Physics Letters $27,068701$.

[15]. S. Nadeem, N.S. Akbar, (2011).Influence of heat and mass transfer on the peristaltic flow of a Johnson Segalman fluid in a vertical asymmetric channel with induced MHD, Journal of the Taiwan Institute of Chemical Engineers 42,58 - 66.

[16]. S. Srinivas, M. Kothandapani, (2009). The influence of heat and mass transfer on MHD peristaltic flow through a porous space with compliant walls, Applied Mathematics and Computation 213, 197-208.

[17]. S. Srinivas, R.Gayathri,(2009). Peristaltic transport of a Newtonian fluid in a vertical asymmetric channel with heat transfer and porous medium, Applied Mathematics and Computation 215, 185-196.

[18]. T. Hayat, S. Noreen, (2010).Peristaltic transport of fourth grade fluid with heat transfer and induced magnetic field, Comptes Rendus Mécanique 338,518 - 528.

[19]. T. Hayat, N. Ali, S. Asghar, Hall effects on peristaltic flow of a Maxwell fluid in a porous medium ,(2007).Physics Letters A 363, 397-403.

[20]. T. Hayat, M.U. Qureshi ,Effect of heat transfer on the peristaltic flow of an electrically fluid in porous space,(2008).Applied Mathematical Modelling 33,1862 - 1873 .

[21]. Y. Abd elmaboud, Kh.S. Mekheimer, (2012). Unsteady pulsatile flow through a vertical constricted annulus with heat transfer, Zeitschrift für Naturforschung $67,185-194$.

[22]. Y.Miyamoto ,M. Hanano, T. Iga,( 1983) Concentration profile in the intes tinal tract and drug absorption model:two-dimensional laminar flow in a circular porous tube, Journal of Theoretical Biology $102,585-601$ 